\title{
Response to the Referee
}

We thank the referee for the helpful and insightful comments and respond as follows.

\section{Major comments}

These comments by the referee are indeed correct and very well taken. Appropriate changes have now been made.

I have the following concerns/questions about this paper:

1. Overlap with [12]: We have restructured the paper. The emphasis is now on the fact that the statistically based approaches to deriving weights from a judgment matrix are shown to emanate quite naturally from the theory underpinning the method of paired comparisons. The subsection on the EVM has been totally removed and some of the sections re-ordered. At the same time we have retained large amounts, indeed most, of the original version. However certain paragraphs which were taken directly from our earlier papers have been reworked. Finally the various methods have been carefully cited.

2. The example in Section 4: The point regarding the example is again very well taken. We have introduced a different small example just to illustrate the methodology and to highlight the Kullback-Leibler distance approach. A comment on the results in relation to comparing the various methods is retained, with some change, in the conclusion.

3. Focus: We hope that we have focussed the paper into a quasi-review, while at the same time tying the methods together within the context of the method of paired comparisons. See our answer to comment 1 as well.

\section{Minor comments}

We have addressed all these as follows:

1. Page: 1, line: 7 There should always be a comma after a the word "however" that starts a sentence. Perform a search for "however" and correct throughout the paper. Done

2. Page: 2, line: 7 Do not use display style mathematics in text lines We have done this and displayed, for example, $\frac{2 \sigma^{2}}{n}$ as $2 \sigma^{2} / n$. 
3. Use the substack command in the amsmath package to stack subscripts for summation signs. Done throughout.

4. Mathematical equations should read as part of sentences and should thus contain commas, full stops, etc. Almost none of the displayed equations contains proper punctuation. Changed throughout.

\section{Small comments}

We have addressed all these as follows:

1. Page: 1, line: 2 "1970s" $\rightarrow$ "1970s" Done.

2. Page: 1, line: 3 Changed.

3. Page: 1, line: 10 Done.

4. Page: 1, line: 11 Point well taken. The claims of "not well-documented" and so on have been removed. Indeed the emphasis of the paper is now rather different.

5. Page: 1, paragraph 2 Avoid one sentence paragraphs. The introduction has been expanded a little so as to explain the structure of the paper.

6. Page: 4, line This has been reworded to make sense.

7. Page: 5, line: 4 This was clumsy. The section on discretization has been cut down and the list is no longer there.

8. Page: 5, line: 8 Done.

9. Page: 6, line: 8 Citation added.

10. Page: 6, line: 12 Citation added.

11. Page: 7, line: 9 All possible values of $\max \left\{a_{i j}, a_{j i}\right\}+1$ on the Saaty scale are $2, \ldots, 10$ and their LCM is 2520.

12. Page: 10, line: 6 The example has been changed. 